\title{
Biofiltration of waste gases in a reactor with a split-feed
}

\author{
JA Mendoza, MC Veiga, C Kennes \\ Journal of Chemical Technology \& Biotechnology, Volume 78, Issue 6 \\ June 2003, Pages 703-708 \\ DOI: $10.1002 /$ jctb.846
}

\begin{abstract}
The efficiency of using different feed strategies was evaluated in the case of a gas-phase biofilter packed with an inert carrier material. During a preliminary control-period, the biofilter was first fed with a single downflow feed of toluene. Reactor performance and biomass distribution were evaluated. The feed was then split into two flows before entering the reactor. Different feed ratios were tested during a 6-month period, following the preliminary control stage. Splitting the feed into equal flow rates through the upper and middle part of the biofilter (in a $50: 50$ ratio) improved the performance compared with the single-feed period. Such a high performance could also be maintained when using a higher flow rate for the upper port than for the middle port, with a feed-ratio of approximately $70: 30$, when more biomass was formed in the upper half of the filter bed. However, performance decreased when inverting this ratio from 70 : 30 to 30 : 70, ie when the highest flow rate was fed through the middle port of the biofilter.
\end{abstract}

\section{Keywords:}

Air pollution control; clogging; downflow biofilter; reactor design; toluene 


\section{INTRODUCTION}

Waste gases have traditionally been treated by means of physical and/or chemical methods based on mass transfer or chemical oxidation. Research undertaken over the past two or three decades has shown the significant potential of microorganisms to degrade a wide range of volatile industrial pollutants. 1 Such potential has recently also been used in biological gas-phase reactors and has given a significant impulse to the development of bioreactors for waste gas treatment to such a level that they have become a widely accepted alternative to the more conventional technologies.2 Besides the conventional biofilter based on the use of natural, usually organic, filter beds, new bioreactors have been developed and alternative biofilters have been proposed, among which one should mention the biotrickling filter and other reactors packed with inert carrier materials.3

Although bioreactors have become reliable systems at an industrial scale, they still need to be further improved and optimized. This is the case for biofilters packed with inert carrier materials. In conventional biofilters based on the use of inert filter beds, an aqueous nutritive solution is only added occasionally compared with biotrickling filters in which an aqueous phase is fed and/or recycled continuously through the system.4 Several possible biomass-related problems or characteristics need to be considered when dealing with such inert carriers. Natural filter beds contain microorganisms and do not necessarily need to be inoculated. However, inert packing materials need always to be inoculated. The selection of an adequate seed culture may affect not only the length of the start-up period5 but also the subsequent maximum performance. 6

The addition of nutrients to inert filter beds is a prerequisite to maintain a high microbial activity and for successful long term biofilter operation. The supply of nutrients and volatile organic pollutants used as carbon and energy sources will lead to biofilm growth. Biomass growth and activity in biofilters packed with inert carriers need to be controlled and optimized. This represents an important recent research topic, which is studied here and described in the present paper. Excess biomass growth is a typical major problem in such bioreactors since it will lead to a high pressure drop and clogging phenomena, eventually generating problems of decreased performance. Different methods have been proposed and evaluated recently, including backwashing, air sparging, the use of specific chemicals and biological predation with either protozoa or mites.7 Another biomass-related problem is the non-homogeneous microbial growth and uneven biodegradation activity along the height of the filter bed.5, 8, 9 It is not unusual to find higher biomass concentrations near the inlet of gas-phase biofilters packed with inert carriers than near the outlet. A similar phenomenon has been reported in the case of biological filters used for waste water treatment.10 Also the nature of the microbial populations present in such biofilters, as well as their biodegradation activity may be different at different reactor heights.5, 8, 9, 11 Under such conditions, the bioreactor might be operating below its optimal performance. 
The purpose of the present research study was to evaluate the potential of using a splitfeed system on the performance and biomass distribution of a gas-phase biofilter fed with toluene-polluted air and packed with an inert carrier material, ie perlite. The performance and biomass distribution of a single-feed downflow biofilter used as control was compared with the results obtained with split-feed systems using different feed ratios.

\section{MATERIALS AND METHODS}

\section{Biofilter operation}

The experiments were performed with a downflow laboratory-scale cylindrical glass biofilter (Fig 1). The reactor was packed with perlite which was previously sieved in order to eliminate dust and small particles of less than $4 \mathrm{~mm}$ diameter. 4 The biofilter contained four equidistant ports, allowing the operation of the reactor with a split-feed. Polluted air was fed either through the upper part of the biofilter or through both the upper and the middle part of the system. The experiments undertaken to evaluate the effect of splitting the feed lasted about 6 months, avoiding problems typical of long term reactor operation which might affect performance after several years or even sooner, such as pressure drop and clogging. A preliminary 2-month single-feed period was used as control. The reactor was inoculated with active biocatalyst obtained from another biofilter treating toluene-polluted air. The feed was prepared by mixing two different air flows, one of which flowed through a thermostated humidification chamber containing water and another smaller one flowing through a bottle containing toluene. Both were combined in a mixing chamber before reaching the bioreactor (Fig 1). All flow rates were measured and regulated by means of flowmeters. The overall flow rate was $0.15 \mathrm{~m} 3 \mathrm{~h}-1$, although it was sometimes split into smaller flow rates depending on the purpose of each specific experiment. A nutrient solution was added periodically, as described elsewhere (Table 1).4 Two $\mathrm{cm} 3$ each of a vitamin solution and a trace mineral solution were added as well, although they do not significantly affect biofilter performance. 4

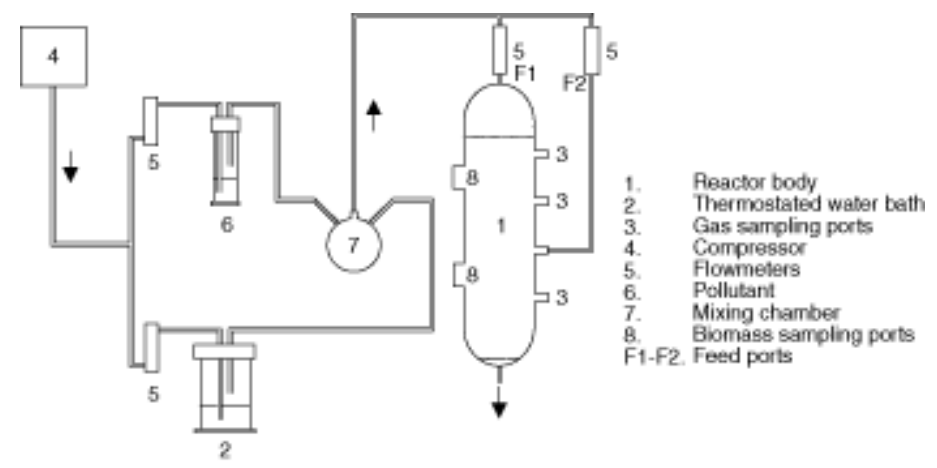

Figure 1.

Experimental set-up with split-feed. 
Table 1. Chemical composition of the nutrient solution added periodically to the biofilter

\begin{tabular}{lc}
\hline Chemical & Concentration $(\mathbf{g ~ d m}-3)$ \\
\hline KH2PO4 & 4.5 \\
K2HPO4 & 0.5 \\
$\mathrm{NH} 4 \mathrm{Cl}$ & 2.0 \\
$\mathrm{MgSO} 4 \cdot 7$ & \\
$\mathrm{H} 2 \mathrm{O}$ & 0.1
\end{tabular}

\section{Analytical methods}

Toluene was measured both at the inlet(s) and outlet of the biofilter. The samples were analyzed on an HP-6890 gas chromatograph (GC) equipped with a flame ionization detector and a 30 m-long capillary HP-5 column (Hewlett-Packard) (5\% phenylmethylsiloxane). The GC was directly coupled to a personal computer for data acquisition. Known amounts of toluene were mixed with air in a carboy. Air samples of different volumes were taken from that carboy and injected in the GC to prepare a calibration curve. The temperature of the injector and the detector was $250{ }^{\circ} \mathrm{C}$ in each case.

The analysis of total solids (TS) and volatile solids (VS) was performed regularly in order to evaluate the growth of biomass in the filter bed. The samples were taken both from the upper and the lower zone of the biofilter. A $2.5 \mathrm{~g}$ filter bed sample was left overnight at $105^{\circ} \mathrm{C}$ for water evaporation and determination of total solids. The sample was then placed in an oven at $550{ }^{\circ} \mathrm{C}$ for $45 \mathrm{~min}$, allowing the calculation of the amount of volatile solids present on the support material.

\section{RESULTS}

\section{Period I-single-feed downward flow}

The biofilter was started-up by feeding it in a conventional downflow mode with a single feed entering through the upper inlet port. Such a system is known to reach its optimal performance usually within a few days after inoculation.5, 6 The present experimental period (period I) lasted 2 months and was used as control, allowing a comparison with the multiple split-feed configurations described below. The 'control' levels of inlet and outlet toluene concentrations are shown in Fig 2. Inlet and outlet concentrations are plotted on the figure rather than the Elimination Capacity versus Pollutant Load since this will allow an easier comparison between the different systems (single-feed and split-feed). The same overall flow rate was maintained throughout the study. Quite high toluene concentrations (around $800 \mathrm{mg} \mathrm{m}-3$ ) were fed immediately 
after seeding the reactor, which reached relatively high removal efficiencies as a result of inoculating an adapted highly active biocatalyst originating from another biofilter treating toluene-polluted air. At the end of this 2-month experimental period, the amount of biomass attached on the packing material was only slightly higher in the upper part of the filter bed than in the lower part of the system (Fig 3, period I), but experience with such biofilters indicates that after long term operation of several months, the amount of biomass increases more strongly near the inlet of the reactor.5

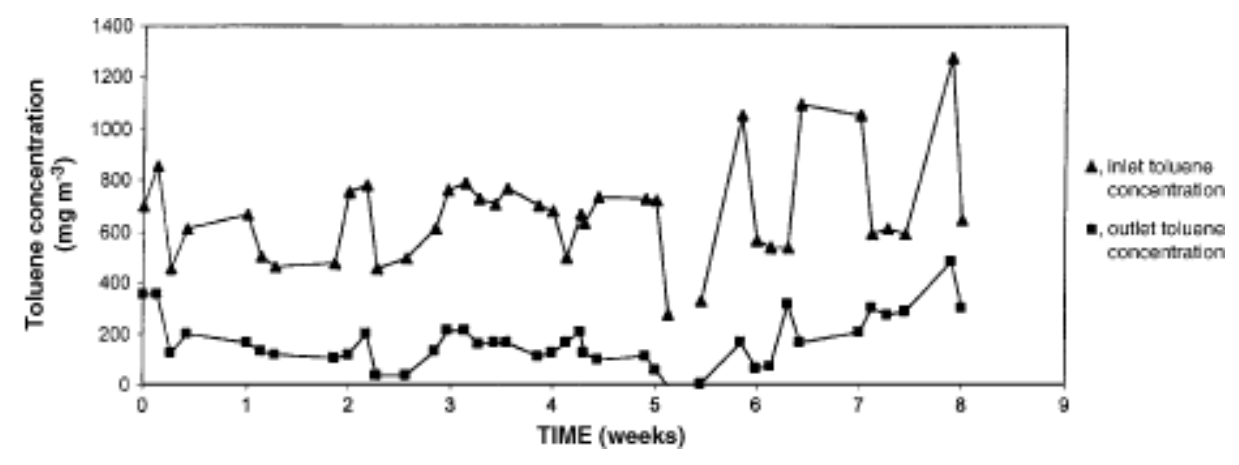

Figure 2. Inlet and outlet toluene concentrations during the control period with the single downflow feed (period I).

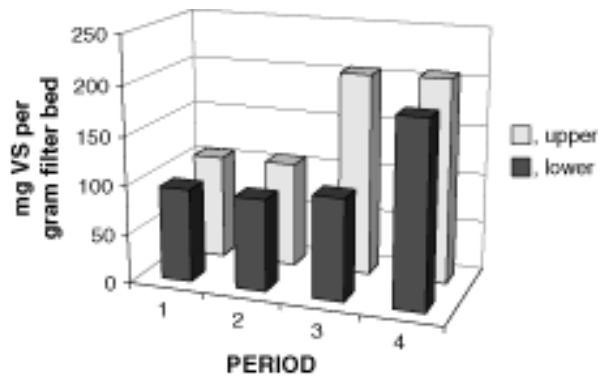

Figure 3. Biomass concentrations in the upper and lower part of the filter bed during: (1) the single-feed, period I; (2) after 26 days of the 50 : 50 split-feed, period II; (3) at the end of the $70: 30$ split-feed, period III; and (4) at the end of the $30: 70$ split-feed, period IV.

\section{Period II-split-feed (50 : 50)}

During the second experimental stage, the polluted air flow was split into two identical flow rates (Fig 1), corresponding each to $50 \%$ of the single flow rate used in the previous experimental step. Thus, around $0.075 \mathrm{~m} 3 \mathrm{~h}-1$ was entered through the upper inlet port of the biofilter and the same amount of polluted air was fed through another inlet port located at the midpoint of the packed bed volume. Toluene concentrations entering the biofilter through both feed ports are represented on Fig 4a. The overall flow rate reaching the biofilter $(0.075 \mathrm{~m} 3 \mathrm{~h}-1+0.075 \mathrm{~m} 3 \mathrm{~h}-1)$ and leaving the system were the same as during the first experimental period $(0.15 \mathrm{~m} 3 \mathrm{~h}-1)$. Unless reaching quite low toluene concentrations (CToluene < KsToluene, in which Ks is the half saturation constant), such a perlite biofilter may be assumed to be operating under plug flow regime with zero order kinetics and reaction limitation.5, 12 Although one single reactor is used, the present split-feed configuration simulates two plug flow reactors in series 
with a side feed to the second reactor. The total volume of these two identical plug flow reactors is the same as the volume of the single-feed biofilter. The design equation is: 3

$R E=\frac{A_{\mathrm{s}} \cdot \delta \cdot h \cdot k_{0}}{U \cdot C_{\ln }}(1)$

where RE represents the Removal Efficiency (\%), As is the biolayer's surface area per volume of filter bed, $\delta$ is the effective biofilm thickness, h is the height of the filter bed, $\mathrm{k} 0$ is the zero order rate constant, $\mathrm{U}$ is the superficial gas velocity and Cin is the influent pollutant concentration. It can easily be shown that the same maximal removal efficiency should theoretically be reached with both systems provided biofilm characteristics and distribution are homogeneous throughout the system. However, recent experimental studies with conventional single-feed biofilters have shown that biomass distribution and characteristics are often not homogeneous and that the biomass concentration and pollutant biodegradation are usually higher nearer the inlet of the biofilter.5, 13, 14 The split-feed (50 : 50) flow pattern should facilitate a more homogeneous distribution of biomass along the height of the filter bed and thus a more optimized biodegradation pattern. Such a hypothesis can indeed be checked and confirmed from the results shown in Fig 4b, since a similar removal efficiency, above $80 \%$, could be reached both during this split-feed period and during the previous singlefeed stage, but significantly higher concentrations of toluene could be fed to the splitfeed biofilter (Fig 4b) than to the single-feed reactor (Fig 2). The highest overall toluene concentration (which takes into account the amount of pollutant fed through both ports) fed occasionally during this period was about $2.5 \mathrm{~g} \mathrm{~m}-3$ with a removal efficiency over $80 \%$ (Fig $4 \mathrm{~b}$ ), while the highest toluene concentration fed during the first experimental period with the single downflow feed was $1.3 \mathrm{~g}$ toluene per $\mathrm{m} 3$ with a removal efficiency of only about 65\% (Fig 2). Removal efficiencies above $80 \%$ were reached during the single-feed experiment at inlet concentrations around $0.8 \mathrm{~g} \mathrm{~m}-3$. The amount of biomass measured after 26 days operation with the split-feed was quite similar in the upper and the lower part of the biofilter (Fig 3, period II). 

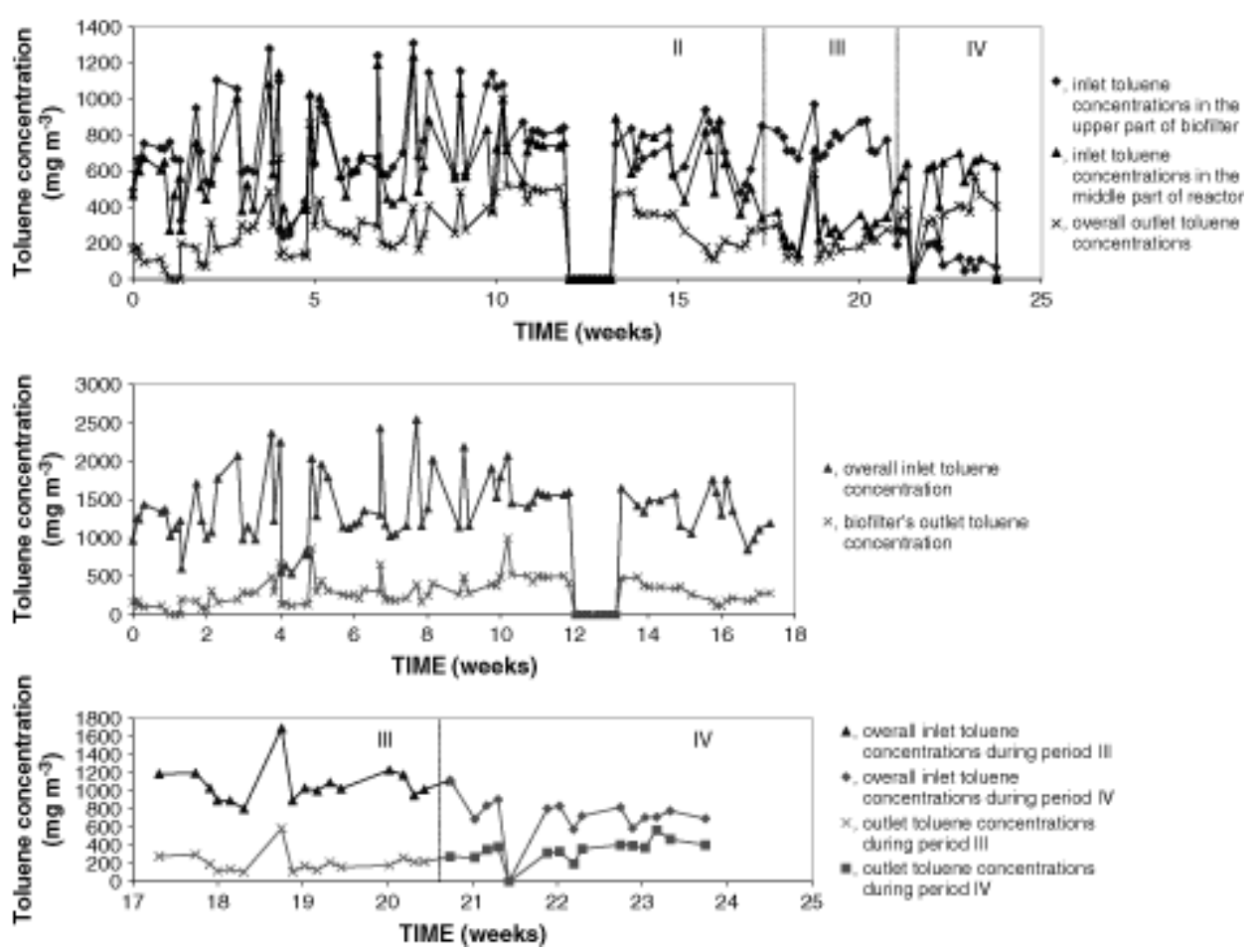

Figure 4. Inlet toluene concentrations in the upper part of the biofilter (F1) and the middle part of the reactor (F2), and overall outlet toluene concentrations, during the three split-feed experimental stages (II, III, and IV). Time zero on the figure corresponds to the first day of the split-feed (50:50) experiment (ie period II), which was preceeded by a 2-month control stage (period I, not plotted). 4b Overall inlet toluene concentrations, including the toluene concentrations entering through both the upper and the middle port, and biofilter's outlet toluene concentration, during period II corresponding to the 50 : 50 split-feed experiment. 4c Overall inlet toluene concentrations during period III (70:30) and period IV (30:70), and outlet toluene concentrations during period III and period IV.

As a result of a technical problem with the air compressor around weeks 12-13 of splitfeed operation, the feed was stopped for approximately one week during that period, although this did not at all affect the biofilter's performance after restoring the normal operating conditions (Figs 4a and 4b). The reactor recovered its original performance immediately after restarting the feed despite the quite high inlet concentration (above $1.5 \mathrm{~g} \mathrm{~m}-3)$.

\section{Period III-split-feed (70 : 30)}

In a third experimental stage, the same two feed ports were used, but $0.10 \mathrm{~m} 3$ polluted air was fed per hour through the upper port of the biofilter while $0.05 \mathrm{~m} 3 \mathrm{~h}-1$ was entering through the middle port of the reactor, corresponding to a percentage flow rate ratio of roughly $70: 30$. Under such operating conditions, a high biofilter performance could still be expected, even if only part of the pollutant is removed in the upper part of the reactor, since the partly treated air stream will still flow through the lowest half of the filter-bed in which it is mixed with an additional, though smaller, flow of 
contaminated air. As shown in Fig 4c, the biofilter's performance was hardly affected by this change in the feed strategy. At overall feed concentrations of $1 \mathrm{~g} \mathrm{~m}-3$ or higher, removal efficiencies above $80 \%$ were reached, which corresponds to similar results as just before switching from the $50: 50$ split-feed to the $70: 30$ feed-ratio (Fig 4c). However, the amount of biomass in the biofilter did proportionally increase more significantly in the upper part of the packing material than in the lower part (Fig 3, period III).

\section{Period IV—split-feed (30 : 70)}

During the last experiment, $0.05 \mathrm{~m} 3 \mathrm{~h}-1$ was fed through the upper port of the biofilter and $0.10 \mathrm{~m} 3 \mathrm{~h}-1$ through the middle port. When modifying the feed ratio from $70: 30$ to $30: 70$, meaning that a proportionally higher flow rate was reaching the biofilter at the level of the middle port than at the upper port, the removal efficiency dropped. Even when feeding overall toluene concentrations below $1 \mathrm{~g} \mathrm{~m}-3$, less than $50 \%$ overall removal efficiency was reached (Fig 4c). This phenomenon results from the fact that the highest fraction of the flow rate, ie $70 \%$, passed through only half the total packed bed volume, giving a too short residence time to allow high pollutant removals. High(er) concentrations of substrate are available in that lower part of the biofilter bed than in the upper part, generating a higher build-up of biomass in the lower part (Fig 3, period IV). But this does not give high removal efficiencies because of the reduced residence time. At the end of this study, a complex bacterial-fungal microbial community was observed under the microscope with the presence of different types of bacteria, yeasts and filamentous fungi.

\section{DISCUSSION}

Gas phase biofilters are very efficient systems for the removal of biodegradable volatile pollutants at concentrations below maximal values of 4-5 g m-3.3 Nevertheless, they still need to be further optimized. One characteristic of such bioreactors packed with inert materials is that they are sometimes operating below their optimal performance level because of a non-homogeneous distribution of biomass and an uneven VOC biodegradation activity along the reactor. Such non-homogeneous distribution has been observed and recently reported by a few authors.4, 5, 9, 11 With a similar perlite-packed biofilter as used in the present work, and fed with an alkylbenzene mixture, approximately $40 \%$ more biomass was found near the inlet of the biofilter than near the outlet.5 Similar results have been reported by other authors with other types of packing materials. Indeed, $\mathrm{Lu}$ et al14 detected up to more than $50 \%$ more biomass near the entrance than near the outlet of a coal-packed biofilter fed with pentane. This phenomenon will lead to faster and higher head losses near the inlet of the reactor, requiring a more frequent application of a biomass control strategy such as backwashing or air sparging7 than when managing obtaining a more homogeneous biomass growth. Not only do different amounts of biomass appear at different filter bed heights, also different microbial populations may appear.5, 11 The dominant growth of non-pollutant degrading populations near the outlet of the bioreactor, where only low concentrations 
of pollutant are available, is not excluded. It is worth mentioning that the biomass concentration does not necessarily always decrease linearly with the height of the filter bed and that some biofilters do sometimes present a near homogeneous biomass distribution.15

An uneven biomass distribution may be explained by the consideration that when high, though non-inhibitory, pollutant concentrations are fed to a biofilter, more substrate will be available near the inlet of the system, resulting in heavier biomass growth in that region. Non-homogeneous biomass distribution may affect both biofilter modeling and reactor performance. Indeed, most classical mathematical models used in gas-phase biofiltration assume that the characteristics of the packed bed and the biofilm remain constant along the filter bed. Concerning the biofilter's performance, a more constant biomass concentration and activity would allow higher removal efficiencies to be reached and the growth of non-pollutant degraders, which contribute to clogging but not to VOC removal, to be reduced. Some authors observed that sometimes up to 80-90\% of the pollutant was removed in the upper half of a biofilter bed in which more biomass was found near the entrance of the reactor, while the other half of the system was needed only to remove the remaining $10-20 \%$ of the volatile organic pollutant.14, $16 \mathrm{~A}$ more even biomass distribution and a more stable reactor operation was reported in the case of using a directionally switching feed, consisting of alternating upflow and downflow feed, rather than an unidirectional system.9 The switching frequency appeared to significantly affect biofilter performance. Another strategy described in the present paper, consists of splitting the feed, yielding interesting positive effects. A similar technique applied to an anaerobic filter treating waste water allowed a more homogeneous biomass distribution to be reached.10 First of all, in the gas-phase biofilter used in the present work biomass was distributed more evenly along the packed bed. Secondly, biofilter performance appeared to improve under such operating conditions. Higher overall concentrations of pollutant could be fed to the bioreactor while maintaining high removal efficiencies. This was true both when splitting the flow rate into two similar flow rates and also when feeding slightly higher flow rates through the upper feed port.

This can be explained since that part of the polluted air entering through the upper port will flow through all the packed bed volume resulting in the highest residence time for that fraction of the polluted stream, compared to the stream entering through the middle inlet port which flows only through half the packed bed volume. Hence, the split-feed strategy appears to have operational advantages.

\section{Acknowledgements}

The present research was supported by projects PR404 E2000/6-0 and PPQ2001-0557. JAM would like to thank CONACyT (México) for the award of a $\mathrm{PhD}$ fellowship. 


\section{REFERENCES}

Van Agteren MH, Keuning S and Janssen DB, Handbook on Biodegradation and Biological Treatment of Hazardous Organic Compounds. Kluwer Academic Publishers, Dordrecht, The Netherlands (1998).

2Dragt AJ, Opening address, in Biotechniques for Air Pollution Abatement and Odour Control Policies, ed by DragtAJ and van HamJ Elsevier Science Publishers, Amsterdam, The Netherlands, pp 3-9 (1992).

3Kennes C and Veiga MC, Bioreactors for Waste Gas Treatment, Kluwer Academic Publishers, Dordrecht, The Netherlands (2001).

4Prado OJ, Mendoza JA, Veiga MC and Kennes C, Optimization of nutrient supply in a downflow gas-phase biofilter packed with an inert carrier. Applied Microbiology and Biotechnology 59: 567-573 (2002).

5Veiga MC and Kennes C, Parameters affecting performance and modeling of biofilters treating alkylbenzene-polluted air. Applied Microbiology and Biotechnology 55: 254-258 (2001).

6Kennes C, Cox HHJ, Doddema HJ and Harder W, Design and performance of biofilters for the removal of alkylbenzene vapors. Journal of Chemical Technology and Biotechnology 66: 300-304 (1996).

$\square$ 7Kennes $\mathrm{C}$ and Veiga MC, Inert filter media for the biofiltration of waste gasescharacteristics and biomass control. Re/Views in Environmental Science \& Bio/Technology 1: 201-214 (2002).

8Smith FL, Sorial GA, Suidan MT, Breen AW, Biswas P and Brenner RC, Development of two biomass control strategies for extended, stable operation of highly efficient biofilters with high toluene loadings. Environmental Science and Technology 30: 1744-1751 (1996).

9Song $\mathrm{J}$ and Kinney KA, Effect of directional switching frequency on toluene degradation in a vapor-phase bioreactor. Applied Microbiology and Biotechnology 56: 108-113 (2001).

10Veiga MC, Pan M, Blázquez R, Méndez R and Lema JM, A double-feed anaerobic filter for the treatment of high strength wastewaters. Biotechnology Techniques 8: 7782 (1994).

11Paca J, Koutsky B, Maryska M and Halecky M, Styrene degradation along the bed height of perlite biofilter. Journal of Chemical Technology and Biotechnology 76: 873878 (2001). 
$\square$ 12Veiga MC, Fraga M, Amor L and Kennes C, Biofilter performance and characterization of a biocatalyst degrading alkylbenzene gases. Biodegradation 10: 169176 (1999).

13Song $\mathrm{J}$ and Kinney KA, Effect of vapor-phase bioreactor operation on biomass accumulation, distribution and activity. Biotechnology and Bioengineering 68: 508-516 (2000).

14Lu C, Lin M-R and Wey I, Removal of pentane and styrene mixtures from waste gases by a trickle-bed air biofilter. Journal of Chemical Technology and Biotechnology 76: 820-826 (2001).

15Cox HHJ, Moerman RE, van Baalen S, van Heiningen WNM, Doddema HJ and Harder W, Performance of a styrene-degrading biofilter containing the yeast Exophiala jeanselmei. Biotechnology and Bioengineering 53: 259-266 (1997).

16Juneson C, Ward OP and Singh A, Microbial treatment of a styrene-contaminated air stream in a biofilter with high elimination capacities. Journal of Industrial Microbiology and Biotechnology 26: 196-202 (2001). 